\title{
Subcutaneous versus intravenous administration of heparin in the treatment of deep vein thrombosis; which do patients prefer? A randomized cross-over study
}

\author{
A.M. Robinson, K.A. McLean, M. Greaves and K.S. Channer \\ Department of Cardiology and Haematology, Royal Hallamshire Hospital, Sheffield S10 2JF, UK
}

\begin{abstract}
Summary: Patient preference for intravenous or subcutaneous heparin in the treatment of deep venous thrombosis was assessed in a randomized cross-over study. Twenty patients with venographically proven deep venous thrombosis were randomized to receive subcutaneous or intravenous heparin for 3 days followed by 3 days of the other treatment. Discomfort at the injection site, assessed by visual analogue scale, was significantly less for the subcutaneous than the intravenous administration route $(P<0.001)$, mobility was thought to be better when receiving subcutaneous heparin $(P<0.005)$ and patients' overall preference was for subcutaneous treatment $(P<0.001)$.
\end{abstract}

\section{Introduction}

The treatment of deep venous thrombosis (DVT) is anticoagulation with heparin. This is usually administered intravenously (IV), but can be given subcutaneously (SC). The efficacy of the two methods of delivery has been assessed in a number of trials which individually have produced conflicting results. However, an overview ${ }^{1}$ suggests that there are no significant differences with respect to death, pulmonary emboli and significant haemorrhage.

Complications can occur with both methods of administration. Intravenous heparin can lead to chemical phlebitis and bacteraemia, ${ }^{1}$ whilst SC heparin is associated with bruising at the site of injection. ${ }^{2}$ It has been suggested that the $\mathrm{SC}$ route is preferable because it reduces the time spent by medical and nursing staff in administration, ${ }^{1}$ and the risk of local complications such as chemical phlebitis and bacteraemia. No study has examined patient preference for either of the treatment modalities. This randomized cross-over study compares the patient acceptability for SC versus IV heparin in the treatment of DVT.

\section{Method}

Twenty patients ( 7 male; mean age 55 years, range 20-85 years) admitted consecutively with a DVT, proven by venogram were entered into the study. Informed written consent was obtained from each patient and the study was approved by the hospital Ethics Committee. Each patient was randomly

Correspondence: K.S. Channer, M.D.

Accepted: 4 September 1992 allocated to receive either calcium heparin SC twice daily (Calciparine, Sanofi, UK) or IV sodium heparin (PumpHep, Leo laboratories, Aylesbury, UK) by mains electric infusion pump. After 3 days the patients received the other treatment with total heparin treatment lasting for 6 days.

When IV therapy was the first arm of treatment a 5,000 unit bolus was given, followed by 30,000 units over 24 hours. The starting dose for subcutaneous heparin was 15,000 units twice daily. Activated partial thromboplastin time (APTT) was measured daily between $0900-1200$ hours whilst IV heparin was administered and at 6 hours after the initial SC injection. The heparin dose was then adjusted to maintain the APTT between 1.5 and two times the upper limit of the control.

On the third day of each treatment period blood samples were taken from all patients 3 hourly for the measurement of APTT and heparin concentration from 0900 for 12 hours. This provided pharmacokinetic data to assess the efficacy of anticoagulation.

The acceptability by the patients of the two methods of administration was assessed using visual analogue scales (VAS), for discomfort in the affected leg, pain at the injection site and mobility. The extremes $(0-100 \mathrm{~mm})$ of the first two scales were marked 'no discomfort' and 'severe discomfort', and the scale for mobility was marked 'fully mobile' and 'bed bound'. Fresh scales were presented to the patients daily, who were also asked to express an overall preference for one or other form of treatment at the end of the study.

Non-parametric statistical analyses were used and $95 \%$ confidence intervals are quoted. A probability of $P<0.05$ was accepted as significant. 


\section{Results}

Nineteen patients completed the study, one being withdrawn because of bleeding whilst receiving IV heparin (APTT within the therapeutic range). The VAS scores were analysed by the Wilcoxon matched pairs signed rank test. When the two methods of administration were compared for all the patients, significantly less discomfort was felt at the injection site (median (range)) for SC heparin (3 mm (0-61 mm)) compared with IV heparin (12 $\mathrm{mm}(0-97 \mathrm{~mm}), P<0.001,95 \%$ confidence intervals for difference $1,15 \mathrm{~mm}$ ).

The patients' perception of mobility was significantly better for the final 4 days of treatment when this was SC heparin $(41 \mathrm{~mm}(0-100 \mathrm{~mm}))$ compared with IV heparin (72 mm $(23-99 \mathrm{~mm})$, $P<0.005,95 \%$ confidence intervals $(12,43 \mathrm{~mm})$. The discomfort felt in the affected leg was no different for either method of treatment $(P=0.54)$.

Heparin and APTT levels were not significantly different for either SC or IV heparin (Figures 1 and 2). The proportion of time for which the APTT was within the therapeutic range was similar to previous studies, ${ }^{1}$ and there was no significant difference between IV (50\%) and SC (46\%).

Fifteen of the 19 patients expressed a preference for the SC route for administration of heparin. Two preferred the IV route and two gave no preference $\left(\chi^{2}=10.5, P<0.001\right)$.

\section{Discussion}

The majority of patients in this study preferred SC to IV heparin in the treatment of uncomplicated deep venous thrombosis. Published trials indicate that the two methods are equally efficient in the prevention of pulmonary emboli and that SC heparin is not associated with an increase in major complications. $^{1-5}$

There are several potential advantages of SC over IV heparin treatment, including a reduction in the time taken to administer, no break in treatment whilst a new heparin infusion is prepared and the

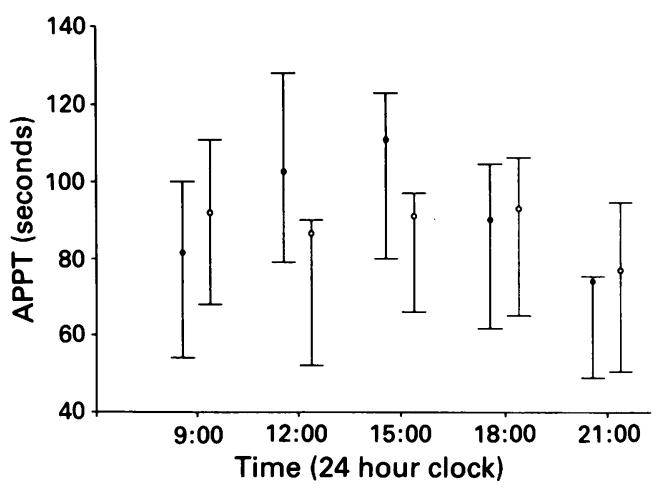

Figure 1 This shows the mean and interquartile range for activated partial thromboplastin time during treatment with SC (O) and IV (O) heparin in 19 patients with deep vein thrombosis on the third day of each treatment period.

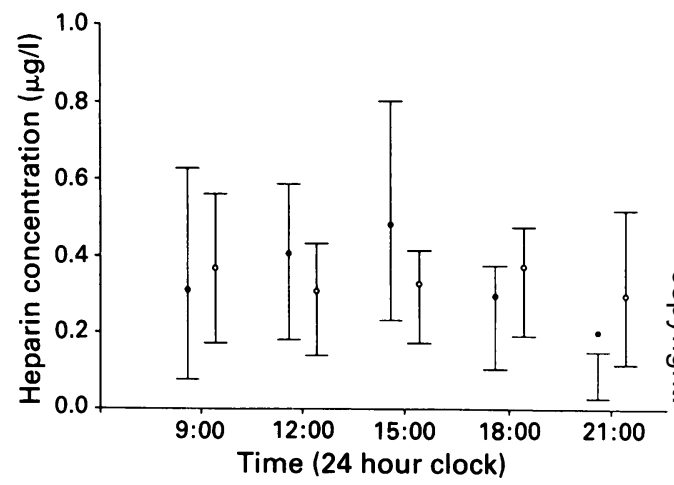

Figure 2 This shows the mean and interquartile range for blood heparin level during treatment with $\mathrm{SC}(\Theta)$ and IV $(O)$ heparin in 19 patients with deep vein thrombosis on the third day of each treatment period.

cost ( $£ 1.98 /$ day for SC heparin compared with $£ 2.30$ /day for IV heparin). Given that there is no difference in efficacy or safety, the most important factor should be patient acceptability. We suggest therefore that SC heparin is the treatment of choice in deep venous thrombosis.

\section{References}

1. Hommes, D.W., Bura, A., Mazzolai, L., Buller, H.R. \& ten Cate, J.W. Subcutaneous heparin compared with continuous intravenous heparin administration in the initial treatment of deep vein thrombosis. A meta-analysis. Ann Intern Med 1992, 116: $279-284$

2. Walker, M.G., Shaw, J.W., Thompson, G.J.L. et al. Subcutaneous calcium heparin versus intravenous sodium heparin in treatment of established acute deep vein thrombosis of the legs: a multicentre prospective randomised trial. $\mathrm{Br} \mathrm{Med} \mathrm{J}$ 1987, 294: 1189-1192.

3. Doyle, D.J., Turpie, A.G.G., Hirsh, J. et al. Adjusted subcutaneous heparin or continuous intravenous heparin in patients with acute deep vein thrombosis. Ann Intern Med 1987, 107 (4): 441 - 445.

4. Andersson, G., Fagrell, B., Holmgren, K. et al. Subcutaneous administration of heparin; a randomised comparison with intravenous administration of heparin to patients with deepvein thrombosis. Thromb Res 1982, 27: 631-639.

5. Bentley, P.G., Kakkar, V.V., Scully, M.F. et al. An objective study of alternative methods of heparin administration. Thromb Res 1980, 18: 177-187. 